\title{
Identification of Dmrt2a downstream genes during zebrafish early development using a timely controlled approach
}

\author{
Rita Alexandra Pinto ${ }^{1}$, José Almeida-Santos ${ }^{1,3}$, Raquel Lourenço ${ }^{1,4}$ and Leonor Saúde $2^{2^{*}}$ (D)
}

\begin{abstract}
Background: Dmrt2a is a zinc finger like transcription factor with several roles during zebrafish early development: left-right asymmetry, synchronisation of the somite clock genes and fast muscle differentiation. Despite the described functions, Dmrt2a mechanism of action is unknown. Therefore, with this work, we propose to identify Dmrt2a downstream genes during zebrafish early development.

Results: We generated and validated a heat-shock inducible transgenic line, to timely control dmrt2a overexpression, and dmrt2a mutant lines.

We characterised dmrt2a overexpression phenotype and verified that it was very similar to the one described after knockdown of this gene, with left-right asymmetry defects and desynchronisation of somite clock genes. Additionally, we identified a new phenotype of somite border malformation.

We generated several dmrt2a mutant lines, but we only detected a weak to negligible phenotype. As dmrt2a has a paralog gene, $d m r t 2 b$, with similar functions and expression pattern, we evaluated the possibility of redundancy. We found that $d m r t 2 b$ does not seem to compensate the lack of $d m r t 2 a$. Furthermore, we took advantage of one of our mutant lines to confirm dmrtza morpholino specificity, which was previously shown to be a robust knockdown tool in two independent studies.

Using the described genetic tools to perform and validate a microarray, we were able to identify six genes downstream of Dmrt2a: foxj1b, pxdc1b, cxcl12b, etv2, foxc1b and cyp1a.

Conclusions: In this work, we generated and validated several genetic tools for dmrt2a and identified six genes downstream of this transcription factor. The identified genes will be crucial to the future understanding of Dmrt2a mechanism of action in zebrafish.
\end{abstract}

Keywords: Dmrt2a, dmrt2a transgenic, Dmrt2b, TALEN mutants, Microarrays, Zebrafish

\section{Background}

The DMRT (Doublesex and Mab3 Related Transcription factors) family of zinc finger like transcription factors has been classically associated with sexual determination and differentiation. Although these functions remain fairly conserved in the different animals studied so far, during evolution different members of this family acquired different functions, such as nervous system development, somitogenesis and left-right asymmetry establishment [1].

\footnotetext{
* Correspondence: msaude@medicina.ulisboa.pt

${ }^{2}$ Instituto de Medicina Molecular e Instituto de Histologia e Biologia do Desenvolvimento, Faculdade de Medicina, Universidade de Lisboa, Avenida Professor Egas Moniz, 1649-028 Lisboa, Portugal
}

Full list of author information is available at the end of the article
When considering the DMRT family, Dmrt2a is particularly notorious because it was the first DMRT protein to be identified with a role apart from sexual determination and differentiation. Meng et al. were the first to describe $d m r t 2 a$ expression pattern in the somites, in zebrafish and mouse, and to propose its role in somitogenesis [2]. Later, $d m r t 2 a$ was found in the Left-Right Organiser (LRO) of zebrafish and chicken, and was found to be necessary not only for somitogenesis but also to the synchronisation of the somite clock genes, left-right asymmetry and more recently, to fast muscle differentiation [3-6]. Moreover, a fish-specific paralog gene $-d m r t 2 b$ - was also identified with similar

(C) The Author(s). 2018 Open Access This article is distributed under the terms of the Creative Commons Attribution 4.0 International License (http://creativecommons.org/licenses/by/4.0/), which permits unrestricted use, distribution, and 
roles regarding somitogenesis and left-right asymmetry establishment $[7,8]$.

In mouse, DMRT2 was only associated with somitogenesis-related processes. It was proposed that this transcription factor could affect extracellular matrix components such as LAMININ-1 and, it was further demonstrated that DMRT2 could act in a PAX3/DMRT2/MYF5 regulatory cascade at the onset of myogenesis $[9,10]$.

On the other hand in zebrafish, several roles were described for Dmrt2a, but little is known about its targets and signalling pathway. Until now, only two players are known to interact with $d m r t 2 a$, at the level of its 3'UTR: Celf1 and miR-203a $[5,6]$. Celf1 is an RNA-binding protein that promotes $d m r t 2 a$ mRNA decay. Upon overexpression of celf1, dmrt $2 a$ mRNA levels are decreased, and problems in the synchronisation of the somite clock genes and in left-right asymmetry establishment arise [5]. miR-203a is a microRNA, which also regulates dmrt $2 a$ mRNA levels, being needed to ensure correct fast muscle development [6].

In this work, our main goal was to identify Dmrt2a downstream genes in order to clarify how this transcription factor controls so many different developmental processes in zebrafish. In order to accomplish this goal, we generated a heat-shock inducible transgenic line that allowed us to induce a timely controlled dmrt2a overexpression, and using the TALEN technology, we generated $d m r t 2 a$ stable mutant lines. Taking advantage of one of the $d m r t 2 a$ mutant lines, we were able to validate the specificity of the previously used $d m r t 2 a$ morpholino
(MO) $[3,5]$. Using our tools, we performed and validated a microarray experiment. We identified foxj $1 b$, $p x d c 1 b, c x c 112 b$, etv2, foxc1b and cyp1a as genes downstream of Dmrt2a. Our findings give the first steps in order to understand Dmrt2a mechanism of action during zebrafish early development.

\section{Results}

Generation of a heat-shock inducible transgenic line to timely overexpress $d m r t 2 a$

We generated a heat-shock inducible transgenic line $\mathrm{Tg}$ (hsp70:HA-dmrt2a) (Fig. 1a) to overexpress $d m r t 2 a$ in a timely controlled manner. We induced a heat-shock (Fig. 1b) at bud-stage given that this time-point coincides with $d m r t 2 a$ early expression (Fig. 1c), marks the beginning of somitogenesis [11] and precedes the formation of zebrafish LRO [12].

To ensure we had a homogenous transgenic population, we selected the fish with only one copy of the transgene (Fig. 1d). We confirmed that after a heat-shock, the HA-Dmrt2a fusion protein was produced with the correct molecular weight (Fig. 1e) and translocated into the nucleus (Fig. 1f). This translocation was controlled by the endogenous nuclear localisation signal contained within Dmrt2a DM domain [13].

\section{Transient increase of $d m r t 2 a$ expression produces a similar phenotype to dmrt2a loss-of-function}

We used the $\operatorname{Tg}(\mathrm{hsp} 70: \mathrm{HA}-d m r t 2 a)$ to investigate the impact of transiently overexpressing $d m r t 2 a$ during the

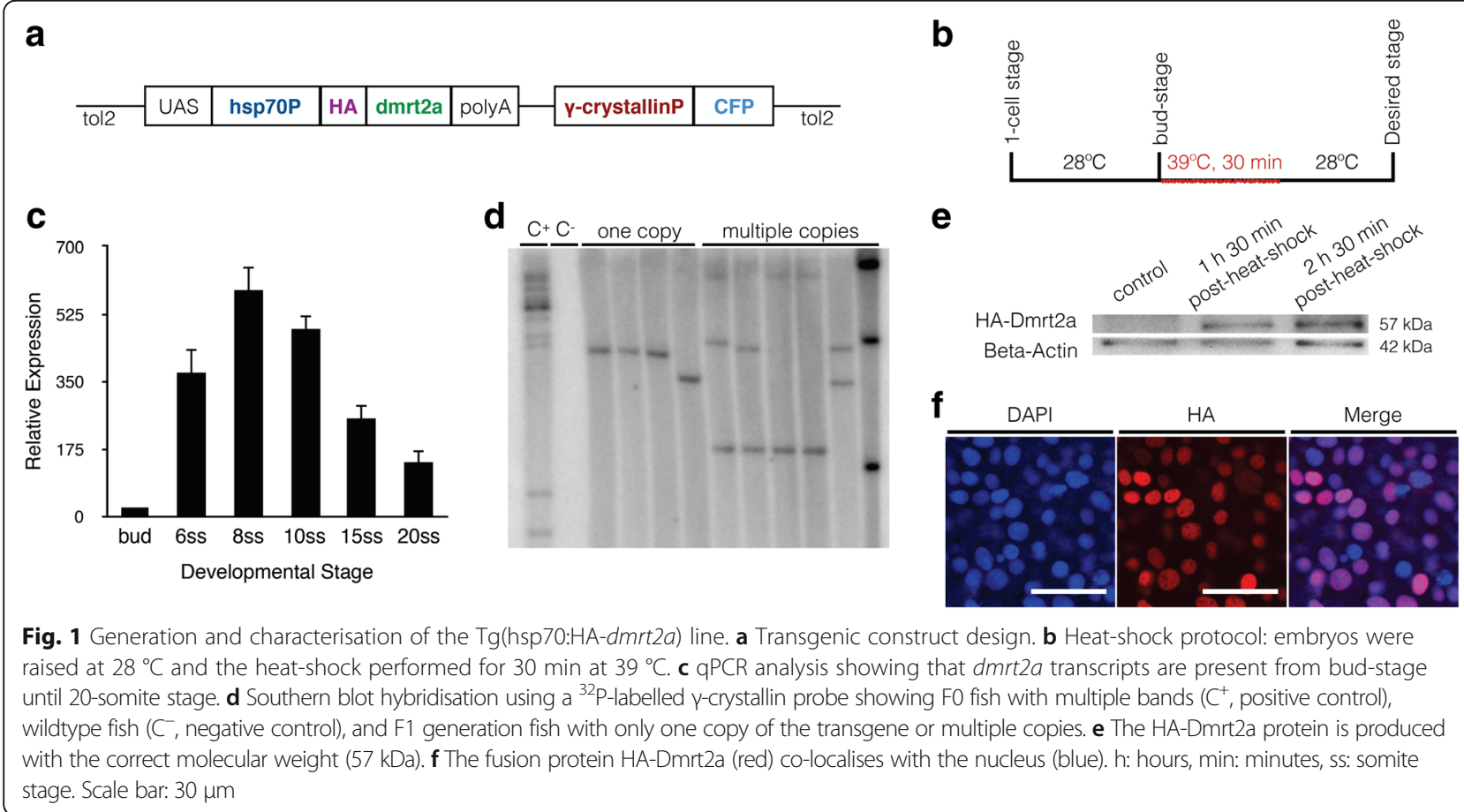


developmental processes known to be regulated by this gene: left-right asymmetry and somite formation $[3,5]$.

In the context of left-right asymmetry establishment, $d m r t 2 a$ overexpression led to an increased bilateral expression of the laterality markers, spaw and pitx2, when compared to controls (Fig. 2a, b). As Nodal signalling promotes the left-jog of the heart tube [14] and given that spaw and pitx2 became bilateral upon $d m r t 2 a$ overexpression, we expected an equivalent percentage of no-jog heart phenotype. However, we obtained an increased percentage of right-jog phenotype, when compared to controls (Fig. 2c). Although bilaterally expressed, small differences in spaw levels, between the left and right sides of the lateral plate mesoderm, could be sufficient to drive an asymmetric heart jogging. During heart tube morphogenesis, jogging to the left side is followed by looping to the right side of the embryo (i.e., D-loop). When compared to controls, we obtained a higher percentage of no-loop and S-loop (i.e., left-sided loop) heart phenotypes after $d m r t 2 a$ overexpression, showing a lack of agreement between jogging and looping (Fig. 2d). These results further support the idea that heart looping can be a Nodal independent mechanism [15].
In the context of bilateral somite formation, we obtained more embryos with a desynchronised expression of the somite clock genes, deltaC, her1 and her7 after dmrt2a overexpression, when compared to controls (Fig. 3a-c). To evaluate the impact of this desynchronisation in somitogenesis, we evaluated $p c d h 8$, mespb, myod 1 and $c b 1045$ expression patterns. All these markers revealed defects in somite morphogenesis (Fig. 4a-d) that can be due to poorly defined or absent intersomitic borders, as we observed (Fig. 4e).

Therefore, dmrt2a overexpression produces a similar phenotype to the one previously described when this gene is knockdown $[3,5]$, suggesting a need to fine tune dmrt2a levels during early development. Additionally, we observed a robust new phenotype in the formation of somite boundaries.

\section{A mild to negligible phenotype is observed in dmrt2a mutants}

In order to produce stable $d m r t 2 a$ mutant lines, we used the TALEN technology (Fig. 5a and Additional file 1: Figure S1a, b). We obtained two different mutants: $d m r t 2 a^{\Delta 100}$, which lacks the 3'end of the 5'UTR, the start codon and part of the first exon spanning a $100 \mathrm{bp}$
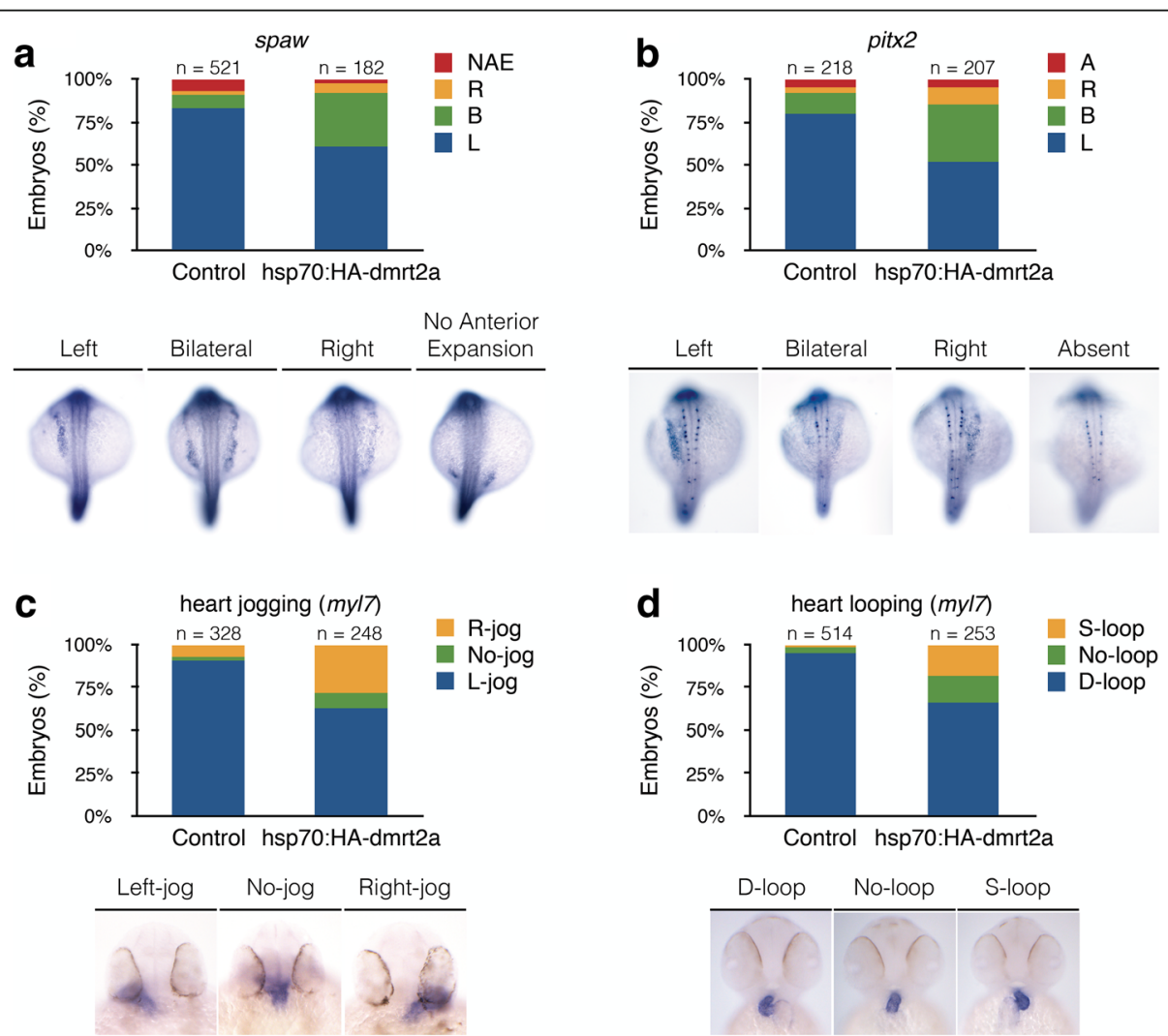

Fig. 2 Characterisation of dmrtza overexpression phenotype: left-right asymmetry. a Left-sided gene spaw (20-somite stage). b Left-sided gene pitx2 (20-somite stage). c Heart jogging at 30 hpf, using a myl7 probe. $\mathbf{d}$ Heart looping at 48 hpf, using a myl7 probe. The embryos shown illustrate the different phenotypes obtained. L: left, R: right, B: bilateral, NAE: no anterior expansion, A: absent, hpf: hours post-fertilisation 

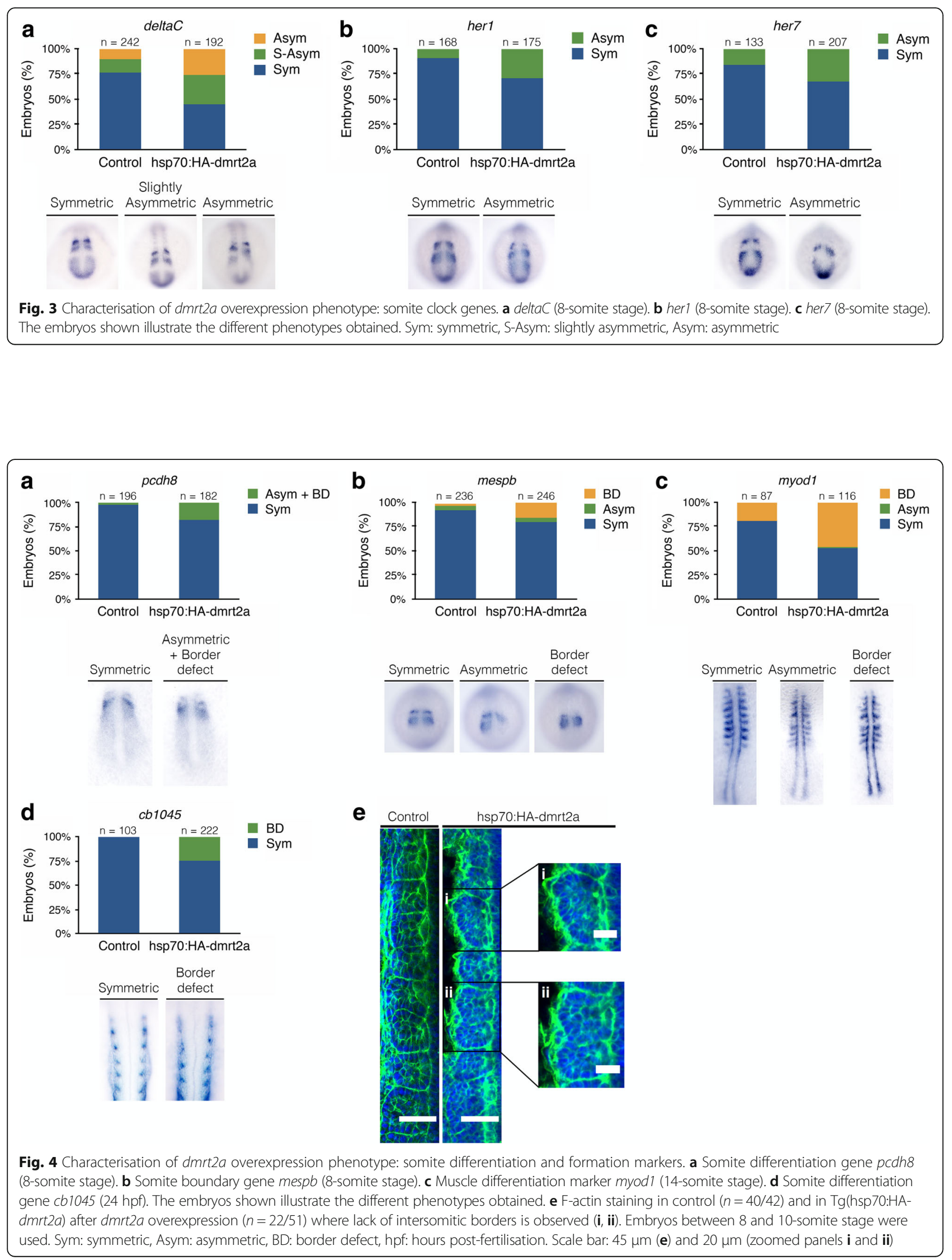


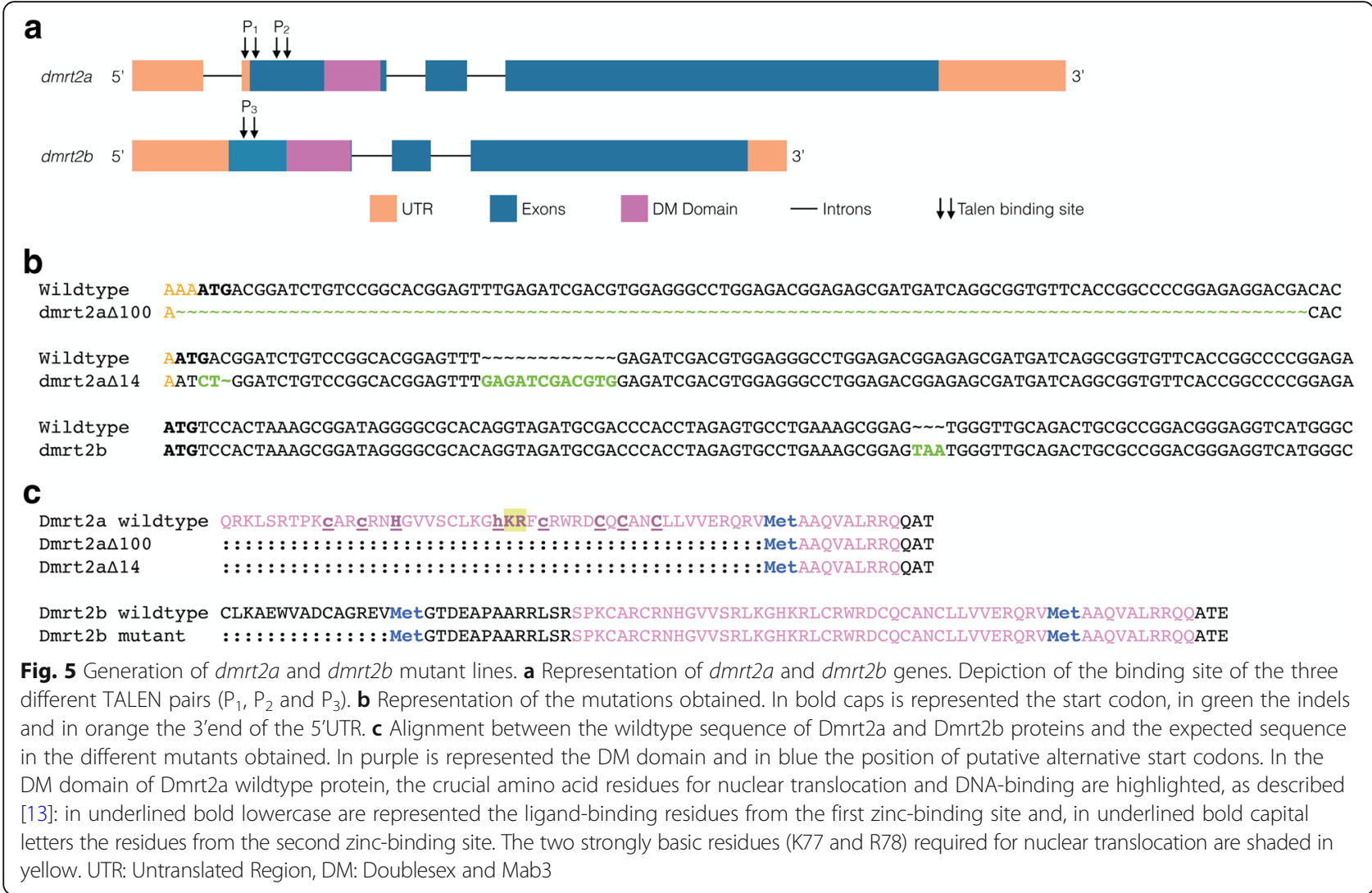

region; and $d m r t 2 a^{\Delta 14}$, which also lacks the start codon and has a 12 bp duplication in the first exon (Fig. 5b). $d m r t 2 a$ mutants in homozygosity were viable and were used to characterise the loss-of-function phenotype. We observed that $d m r t 2 a^{\Delta 14-/-}$ had a mild phenotype regarding spaw expression pattern and $d m r t 2 a^{\Delta 100-/-}$ only had a very mild phenotype regarding heart jogging (Fig. 6a, b). In both cases, her7 expression pattern was unaffected (Fig. 6c).

As $d m r t 2 a$ has a paralog gene - dmrt $2 b$ - with a similar expression pattern in somites and with described roles in somitogenesis and left-right asymmetry establishment $[7,8]$, we wanted to verify if, in the absence of $d m r t 2 a, d m r t 2 b$ could compensate its lack. Therefore, using the TALEN technology, we also produced a $d m r t 2 b$ mutant, which has an early stop codon (p.E22_W23insX, Fig. 5a, b and Additional file 1: Figure S1c). Also in homozygosity, $d m r t 2 b$ mutants were viable and presented an even milder phenotype than $d m r t 2 a$ mutants in the different evaluated markers (Fig. 6a-c). We proceeded with the generation of double mutants: $d m r t 2 a^{\Delta 100} ; d m r t 2 b$ and $d m r t 2 a^{\Delta 14} ; d m r t 2 b$. When analysing the double mutants, we observed that: (1) the spaw phenotype in $d m r t 2 a^{\Delta 14-/-} ; d m r t 2 b^{-/-}$was milder than in the single $d m r t 2 a^{\Delta 14-1-}$ mutant, and (2) the heart jogging phenotype present in $d m r t 2 a^{\Delta 100-/-}$ single mutant, disappeared in the double mutant context (Fig. 6d, e). In both cases, myod1 expression pattern was unaffected (Fig. 6f). As the left-right asymmetry phenotype observed was not consistent between single and double mutants and, as previously reported, zebrafish wildtype embryos can have up to $10 \%$ left-right asymmetry defects [16], we considered our phenotypes negligible.

The weak or negligible phenotype observed in our mutant lines can have several explanations, such as the presence of alternative ATGs, the production of Dmrt2 proteins with residual function or the activation of compensatory genes.

Regarding the first hypothesis, we evaluated all the possible alternative ATGs present from the 5'end of the 5'UTR of $d m r t 2 a$ until the 3'end of the DM domain, which contains the two DNA-binding domains and the crucial amino acid residues for nuclear translocation (Fig. 5c) [13]. We were able to identify only one ATG in dmrt2a mutants, located near the 3'end of the DM domain that maintained $d m r t 2 a$ reading frame (Fig. 5c). If this ATG was used, the DM domain would be truncated and consequently, would lack the residues shown to be crucial for nuclear translocation. As Dmrt2a is a transcription factor, we would expect that the inability to enter the nucleus would render this protein dysfunctional. Regarding $d m r t 2 b$ mutants, we were able to find an alternative 

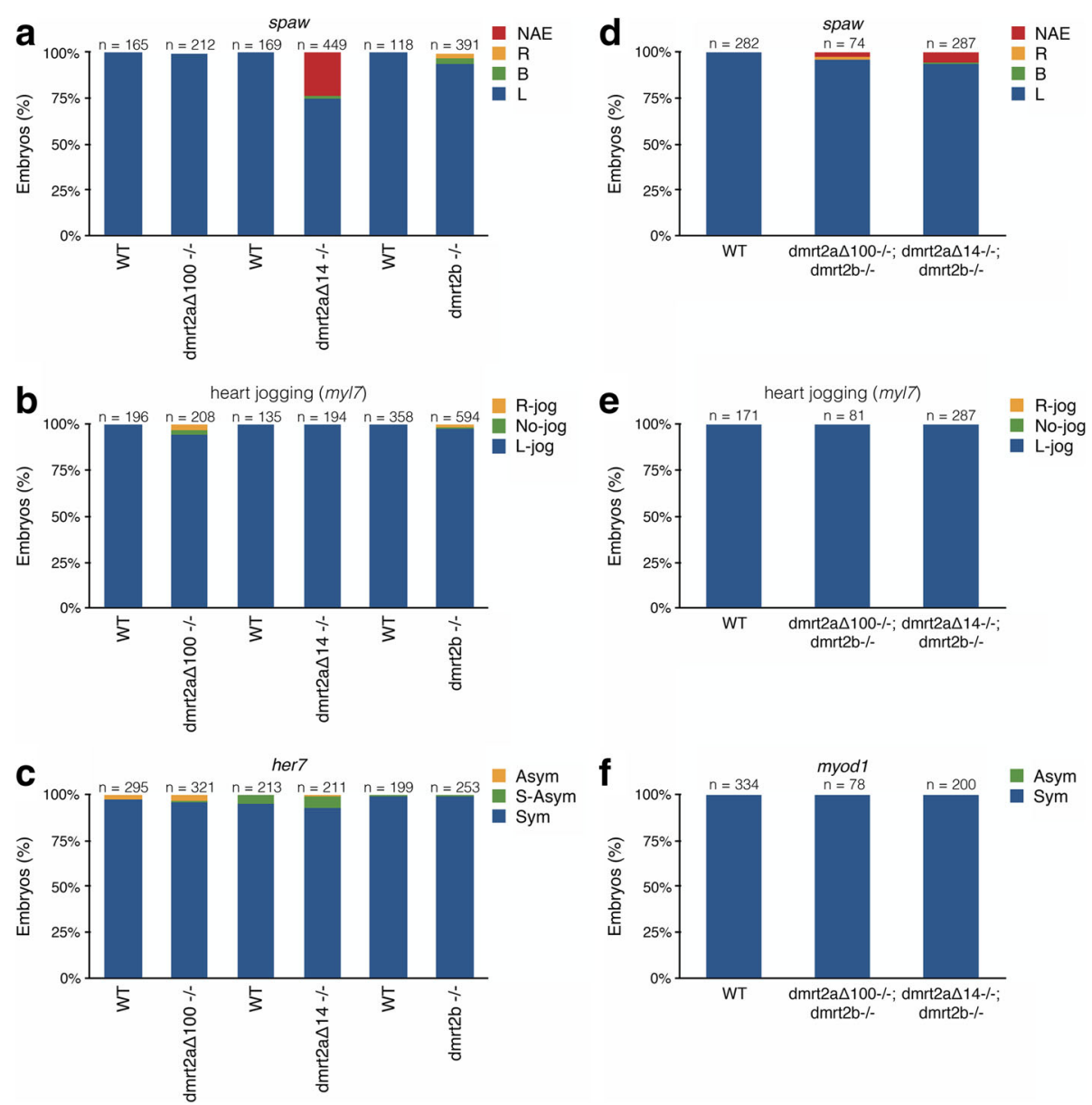

Fig. 6 Characterisation of the single and double $d m r t 2 a$ and $d m r t 2 b$ mutants phenotype. $\mathbf{a}, \mathbf{b}, \mathbf{c}$ Characterisation of $d m r t 2 a^{\Delta 100-/-}, d m r t 2 a^{\Delta 14-/-}$ and $d \mathrm{mrt}_{2} \mathrm{~b}^{-1-}$ mutant phenotype using: (a) left-sided gene spaw (20-somite stage), (b) myl7 for heart jogging at 30 hpf and (c) her7, a cycling

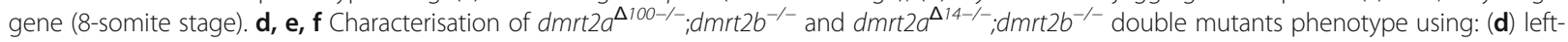
sided gene spaw (20-somite stage), (e) myl7 for heart jogging at 30 hpf and (f) myodl, a muscle differentiation marker (14-somite stage). L: left, R: right, B: bilateral, NAE: no anterior expansion, Sym: symmetric, S-Asym: slightly asymmetric, Asym: asymmetric, WT: wildtype, hpf: hours post-fertilisation

ATG, after the premature STOP codon, which enabled the production of a functional DM domain (Fig. 5c). However, there is no guarantee that this ATG is in an appropriate genomic environment to allow its use.

In the absence of specific antibodies against Dmrt2a and Dmrt2b to evaluate the production of these proteins, we cannot be sure that we have true null mutants. Therefore, we used an indirect approach: we evaluated the levels of both transcripts based on the assumption that, if $d m r t 2 a$ and $d m r t 2 b$ transcripts are dysfunctional they should be degraded.

The results we obtained from this evaluation were the following: (1) in single dmrt2a mutants and in double mutants, dmrt2a transcript was increased; (2) in single $d m r t 2 b$ mutants, its transcript was increased and $d m r t 2 a$ transcript was unaffected; (3) in single $d m r t 2 a$ mutants and in double mutants, $d m r t 2 b$ transcript was not affected $\left(d m r t 2 a^{\Delta 100-/-}\right.$ and $\left.d m r t 2 a^{\Delta 100-/-} ; d m r t 2 b^{-/-}\right)$or was only slightly affected (dmrt2 $a^{\Delta 14-/-}$ and $d m r t 2 a^{\Delta 14-/-} ; d m r t 2 b^{-\prime}$ $\left.{ }^{-}\right)$(Fig. 7). These results show that $d m r t 2 a$ and $d m r t 2 b$ transcripts are under a negative feedback loop regulating their own expression, which suggests a deficiency in the production of Dmrt2a and Dmrt2b proteins. However, we still cannot assume that the mutations obtained produce null alleles. Our data also corroborates the lack of redundancy between $d m r t 2 a$ and $d m r t 2 b$, further supporting previously published results [8].

\section{dmrt2a-MO proves to be specific and non-toxic}

At this moment, we lack specific antibodies to confirm if the different mutants obtained are true nulls and, assuming that a compensatory mechanism is taking place, we have yet to identify possible candidate gene(s) for this role. Nevertheless, we took advantage of one of our mutant lines to re-validate $d m r t 2 a-\mathrm{MO}[3,5]$. 


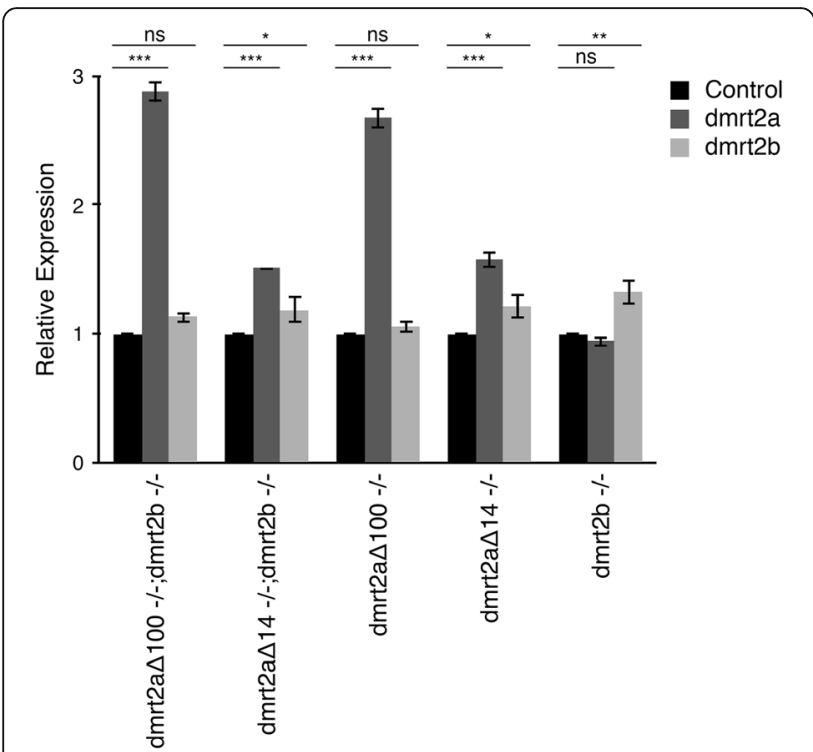

Fig. 7 Evaluation of dmrt2a and $d m r t 2 b$ transcript levels in single and double mutants at $24 \mathrm{hpf} .{ }^{*} P<0.05$, ${ }^{* *} P<0.01$, ${ }^{* *} P<0.001$, ns: not significant. $P$-values were generated using a two-tailed $t$-test. Hpf: hours post-fertilisation

To accomplish this, we used the $d m r t 2 a^{\Delta 100-/-}$ background, which lacks 13 nucleotides of the dmrt2a-MO binding site (Fig. 8a). As the morpholino lacks it preferred binding site, it is the ideal situation to evaluate if it can bind non-specifically to other regions in the genome. To perform this evaluation, we injected dmrt $2 a-\mathrm{MO}$ in the mutant background and compared it with wildtype embryos injected in the same conditions. After injecting $d m r t 2 a-\mathrm{MO}$ in a wildtype background, we observed the expected phenotype in myod 1 and spaw expression (Fig. 8b, c). In contrast, this phenotype was absent or extremely reduced when injecting dmrt $2 a-\mathrm{MO}$ in the mutant background (Fig. 8b, c). Our results suggest that, in the evaluated conditions, dmrt $2 a-\mathrm{MO}$ does not have detectable off-target effects. Additionally, we observed that the toxicity marker - p53 - was not significantly affected after $d m r t 2 a-\mathrm{MO}$ injection (Figs. $8 \mathrm{~d}$, d' and 9b, c for procedure details). As dmrt2a-MO seems to be specific, without off-targets and non-toxic, we decided to use it as a preferential loss-of-function tool.

\section{Genome wide approach to identify Dmrt2a downstream genes}

In the context of the $\operatorname{Tg}($ hsp70:HA-dmrt $2 a)$, the HA-Dmrt2a fusion protein peaks between $1 \mathrm{~h} 30 \mathrm{~min}$ and $2 \mathrm{~h}$ post-heat-shock (Additional file 2: Figure S2). Given that our goal was to identify Dmrt2a most immediate downstream genes we decided to collect embryo samples between $2 \mathrm{~h}(\sim 3$-somite stage $)$ and $2 \mathrm{~h} 30 \mathrm{~min}$ $(\sim 4$-somite stage) after heat-shock (Fig. 9a). Embryo samples were collected using a microdissection protocol. We microdissected Region 1 (containing somites) and
Region 2 (containing tailbud/LRO) which are $d m r t 2 a$ expressing territories (Fig. 9c). These samples were used in a microarray analysis.

In the microarray data (Fig. 9d, e and Additional file 3: Figure S3a, b), dmrt2a levels are increased as expected. However, $d m r t 2 a$ is significantly increased in Region 2 (Fold change $(\mathrm{FC})=2.63, P=0.000008$, False Discovery Rate $(\mathrm{FDR})=0.0212)$ but not in Region $1(\mathrm{FC}=1.3, P=0.000416$, $\mathrm{FDR}=0.0677$ ). This could be due to the higher physiological expression levels of $d m r t 2 a$ in somites (Region 1) when compared to tailbud/LRO (Region 2).

DMRT proteins were described to act as activators, repressors or both [17, 18]. Our microarray analysis revealed that Dmrt2a seems to act mainly as a repressor during early development.

The analysis of the microarray showed some genes that could be expected to be downstream of Dmrt2a, such as myf5 (myogenesis related [10]) and foxj1b (left-right asymmetry related [19]). Interestingly, a category of genes that was revealed to be potentially downstream of Dmrt2a, is related to cardiovascular development (Additional file 4: Figure S4a, b).

We proceeded to validate the microarray data by qPCR. All the selected genes were independently validated, except for ctslb in Region 1 (Fig. 10a, a' and Additional file 5: Figure S5a, a').

\section{Validation of six genes downstream of Dmrt2a: foxj1b, pxdc1b, cxcl12b, etv2, foxc1b and cyp1a}

To ensure that the selected genes were not a result of $d m r t 2 a$ misexpression, we determined their level of expression in a knockdown context, using the previously validated $d m r t 2 a-\mathrm{MO}$. In this combinatorial approach, only the genes down-regulated in the gain-of-function and up-regulated in the loss-of-function, or vice-versa, would be considered to be truly downstream of Dmrt2a activity.

In embryos injected with control-MO or $d m r t 2 a-\mathrm{MO}$, we microdissected Region 1 and Region 2 (Fig. 9b, c) and did qPCR of the previously validated genes (Fig. 10b, b' and Additional file 5: Figure S5b, b').

Using this strategy, we were able to validate five genes: foxj1b, pxdc1b, cxcl12b, etv2 (Region 1) and cyp1a (Region 2) (Fig. 10a-b', for an illustration of the expression pattern of these genes see Additional file 6: Figure S6A1-J2, and for dmrt2a expression pattern see Additional file 7: Figure S7A1-B2'). To ensure that the change of the expression levels of the five selected genes was not due to dmrt2a-MO off-target effects, we injected this $\mathrm{MO}$ in the mutant background and compared it with wildtype embryos. We analysed these embryos using in situ hybridisation and showed that these genes are specifically affected by $d m r t 2 a-\mathrm{MO}$ (Additional file 7: Figure S7C1-C3' and Additional file 8: Figure S8A1-E3). We also observed that similarly to 


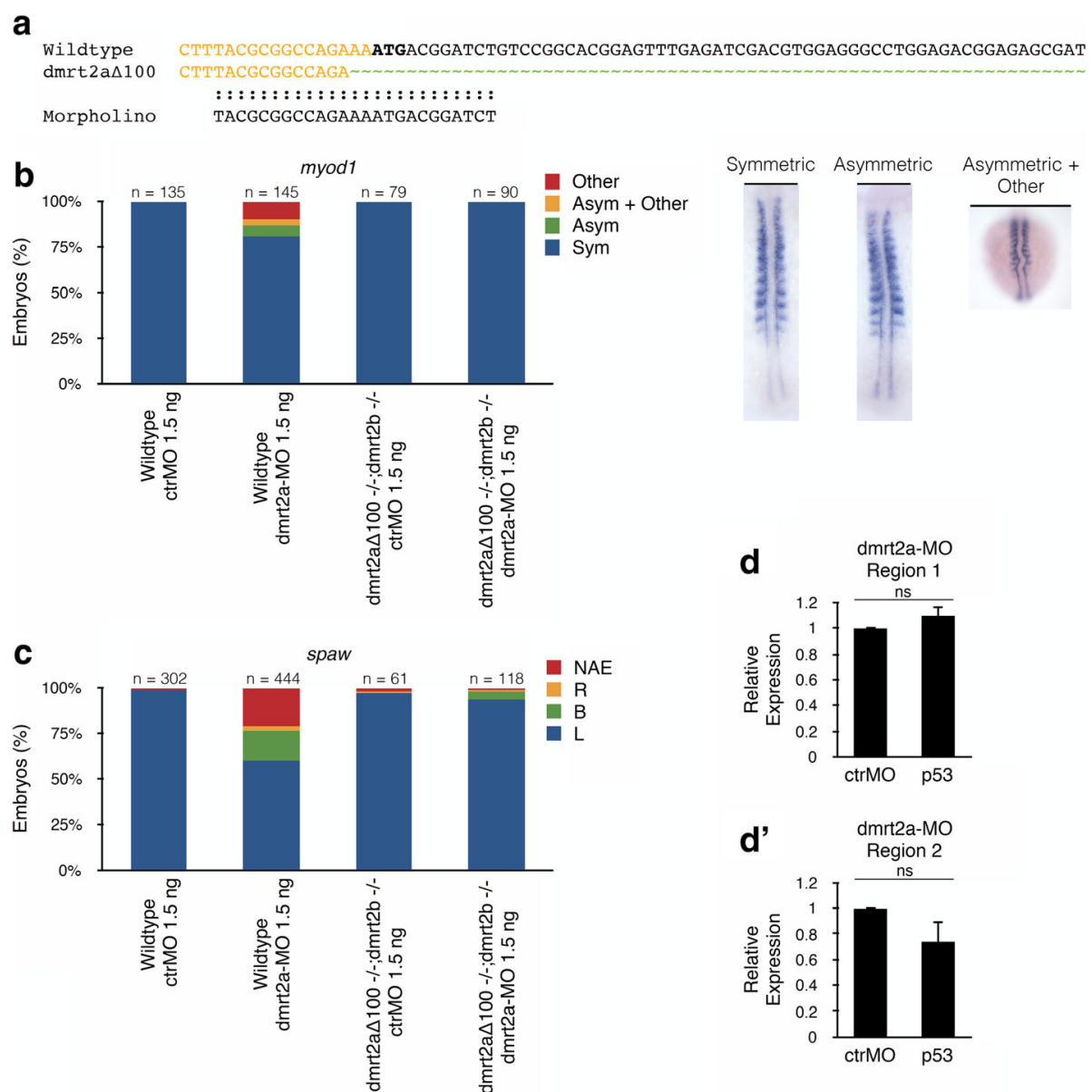

Fig. 8 Evaluation of the specificity of dmrt2a-MO. a Alignment between wildtype dmrt2a sequence, $d m r t 2 a^{\Delta 100}$ sequence and $d m r t 2 a-M O$. The $\mathrm{MO}$ is represented in the sense orientation. In bold caps is represented the start codon, in green the indels and in orange the $3^{\prime}$ end of the $5^{\prime} \mathrm{UTR}$. b, $\mathbf{c}$ Validation of the specificity of dmrt2a-MO using (b) myod1 (14-somite stage) and (c) spaw (20-somite stage). The embryos shown illustrate the different phenotypes obtained in myod1 expression. d, d' Evaluation of p53 levels by qPCR in (d) Region 1 (somites) and (d') Region 2 (tailbud/LRO) after injecting dmrt2a-MO in wildtype embryos. UTR: Untranslated Region, L: left, R: right, B: bilateral, NAE: no anterior expansion, Sym: symmetric, Asym: asymmetric, ctrMO: control morpholino, ns: not significant. $P$-values were generated using a two-tailed $t$-test

what happens in $d m r t 2 a$ mutants, in morphant embryos, $d m r t 2 a$ transcripts are also up-regulated (Figs. 7 and 10b, Additional file 5: Figure S5b and Additional file 7: Figure S7A1-A2').

As Etv2 is a transcription factor with a well-described interaction with the Foxc proteins [20, 21], we verified the presence or absence of Foxc proteins in the microarray data. We found that the zebrafish homolog genes of the mouse Foxc1 - foxcla and foxc1b - were present in the microarray data but with low fold changes $(\mathrm{FC}=-1.04$, $P=0.219, \mathrm{FDR}=0.636 ; \mathrm{FC}=-1.3, P=0.00134, \mathrm{FDR}=0.103$, respectively) (Additional file 3: Figure S3a). While foxcla could not be validated by the loss-of-function approach, we verified that foxc1b was indeed down-regulated after $d m r t 2 a$ overexpression and up-regulated after $d m r t 2 a$ loss-of-function (Fig. 10c, c', for an illustration of the expression pattern of $f \circ x c 1 b$ see Additional file 6: Figure S6 K1-L2'). To ensure that the change of the expression levels of foxc1b was not due to $d m r t 2 a-\mathrm{MO}$ off-target effects, we injected this $\mathrm{MO}$ in the mutant background, as before. We analysed these embryos using in situ hybridisation and showed that $f \circ x c 1 b$ is specifically affected by $d m r t 2 a-\mathrm{MO}$ (Additional file 8: Figure S8F1-F3').

In this work, we identified and validated six genes downstream of Dmrt2a during early development: foxj1b, pxdc1b, cxcl12b, etv2, foxc1b and cyp1a. The relationship between them and Dmrt2a, and their contribution to the different Dmrt2a functions, will be the subject of future studies.

\section{Discussion}

In order to establish the mechanism of action of Dmrt2a, one needs to identify its downstream genes. In this work, we generated and validated several genetic tools that allowed us to identify six genes downstream of 


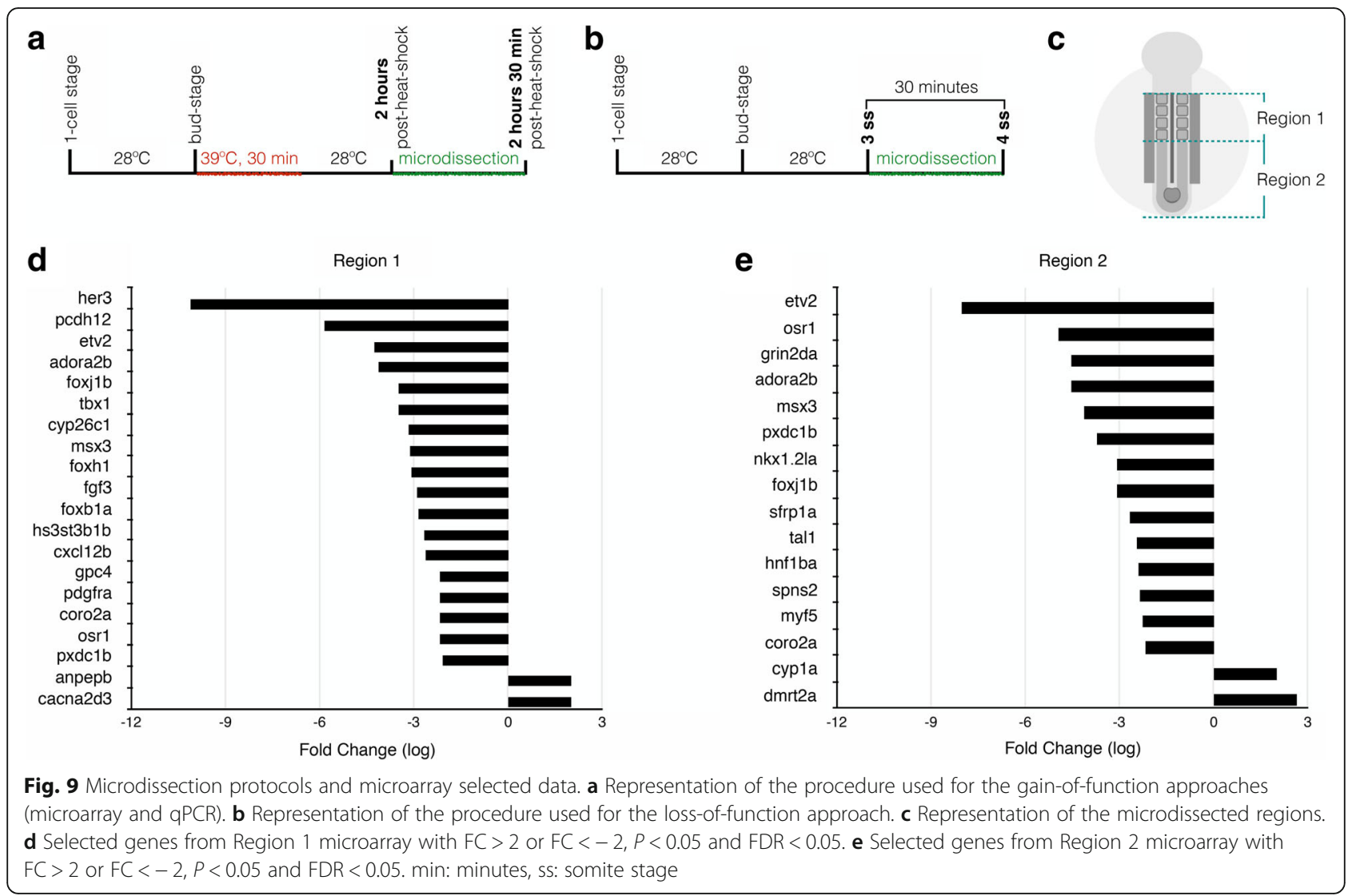

Dmrt2a during early embryonic development: foxj1b, $p x d c 1 b, c x c l 12 b$, etv 2, foxc $1 b$ and cyp $1 a$.

To develop one of our genetic tools - stable $d m r t 2 a$ mutant lines - we used the TALEN technology. However, when considering the different $d m r t 2 a$ mutant lines we generated, we were surprised to detect only a very weak or negligible phenotype, when compared to the previously published morpholino data $[3,5,8]$. The robustness of $d m r t 2 a$ knockdown phenotype had been previously established in two independent studies $[3,5]$, especially in the work of Matsui et al. [5], and its specificity was further confirmed with our work.

The weak phenotype obtained could not be due to the maternal contribution of $d m r t 2 a$ transcripts because we always used an incross between homozygous mutant fish. The presence of alternative ATGs could be an issue, as discussed before, mainly in the case of $d m r t 2 b \mathrm{mu}-$ tant. Another possibility for the weak mutant phenotype is the presence of compensatory gene(s). Our data indicate that $d m r t 2 b$ does not seem to compensate for the lack of $d m r t 2 a$, supporting the findings of Liu et al. [8]. Therefore, to uncover if a compensatory mechanism is taking place, a genome wide approach could be performed comparing mutants and morphants.

To prove the absence of mutant proteins we need specific antibodies that are not available. Thus, in an attempt to understand the outcome of our mutations, we evaluated $d m r t 2 a$ and $d m r t 2 b$ transcripts in the different mutant backgrounds. In theory, if the transcripts are not functional they should be degraded. This hypothesis should have been true at least in the case of $d m r t 2 b$ mutant, due to non-sense mediated decay. However, we observed that $d m r t 2 a$ and $d m r t 2 b$ transcripts are up-regulated in the different mutants evaluated. This suggests that, in the absence of a completely functional transcript/protein, the stimulus to constantly produce more transcript remains, further suggesting that Dmrt2a and Dmrt2b proteins, if produced, should not be completely functional. This does not exclude the possibility of degradation of the mutant transcripts, only that the rate of transcript degradation is slower than the rate of production.

Regarding future approaches to this problem, an effort should be placed in the identification of $d m r t 2 a$ and $d m r t 2 b$ promoter regions, in the generation of bona fide null mutants and in the production of specific antibodies.

As we have no certainty that $d m r t 2 a$ mutants are nulls, we decided against using these lines in the microarray approach. Yet, we were able to take advantage of them to validate $d m r t 2 a-\mathrm{MO}$, confirming its specificity. Therefore, we performed the microarray with $\operatorname{Tg}($ hsp70:HA-dmrt $2 a$ ) and validated its data using $d m r t 2 a-\mathrm{MO}$. 

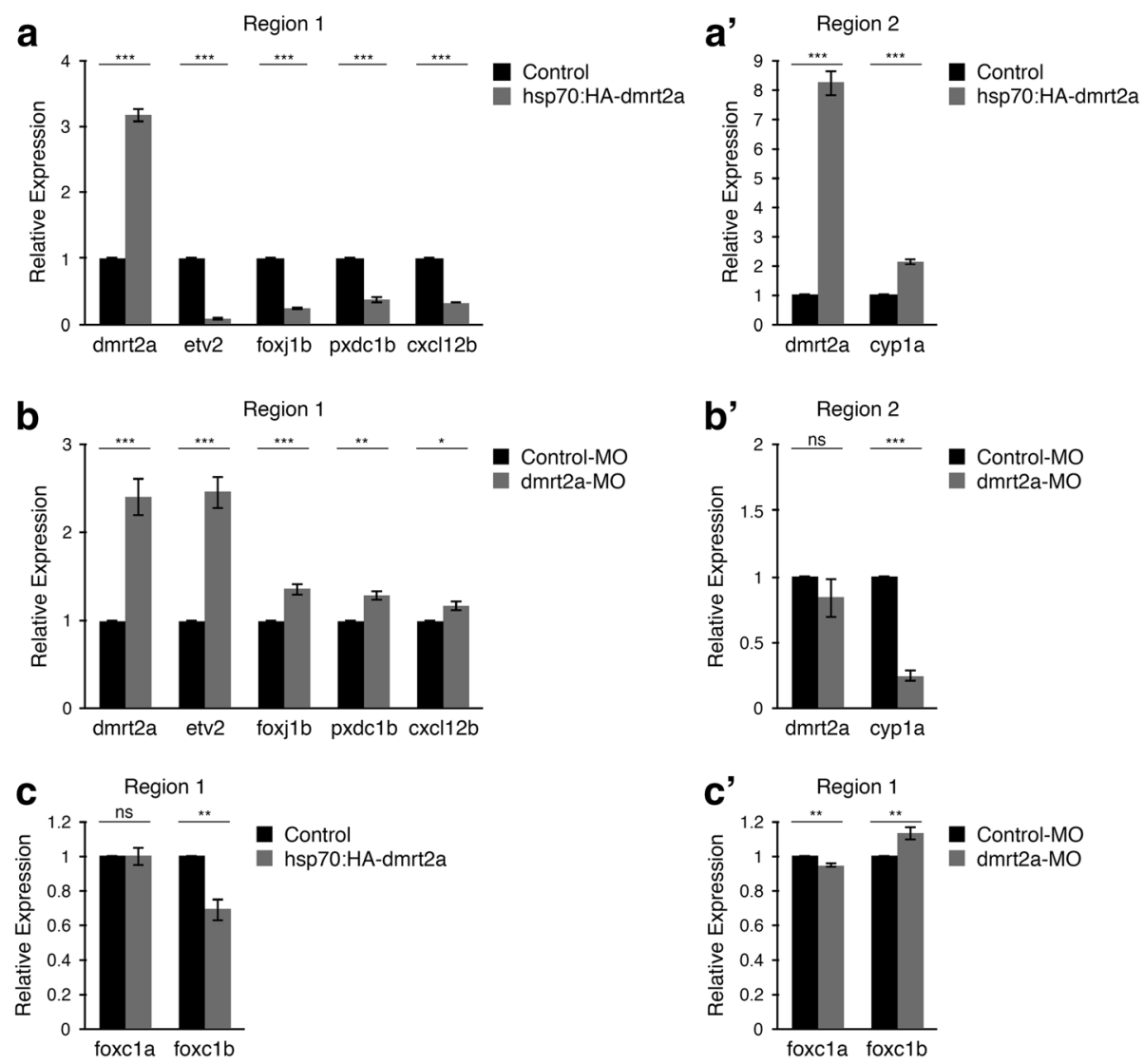

Fig. 10 Microarray data validation. a, a' qPCR validation of selected genes from Region 1 (a) and Region 2 ( $\mathbf{a}^{\prime}$ ), in a gain-of-function context. b, $\mathbf{b}^{\prime}$ qPCR of selected genes from Region 1 (b) and Region 2 ( $\mathbf{b}^{\prime}$ ), in a loss-of-function context. c, $\mathbf{c}^{\prime}$ Evaluation of foxcla and foxc1b transcripts by qPCR using Region 1 samples, in gain-of-function (c) and loss-of-function ( $\mathbf{c}^{\prime}$ ) experiments. ${ }^{*} P<0.05,{ }^{*} P<0.01,{ }^{* *} P<0.001$, ns: not significant. $P$-values were generated using a two-tailed $t$-test

From the list of microarray validated genes, foxj1b [19] is a strong candidate to mediate the left-right asymmetry phenotypes of Dmrt2a, which were previously described in gain and loss-of-function approaches.

With our timely controlled $d m r t 2 a$ overexpression experiments we identified a new phenotype in somite border formation, which recalls the Dmrt2 mouse mutant phenotype [9]. The defects observed in the mouse mutants were associated with a strong reduction of the levels of LAMININ-1, an extracellular matrix protein [9]. While in our pool of six validated genes, we did not identify classic extracellular matrix related genes, we found an interesting candidate, $p x d c 1 b$. Pxdc1b belongs to a family related to membrane attachment to organelles of the endocytic and secretory systems via binding of phosphoinositide lipids [22]. As extracellular matrix proteins are continuously being secreted [23], $p x d c 1 b$ could establish a link between Dmrt2a, extracellular matrix deposition and somite border defects.

Additionally, in our microarray, we identified cyp1a as a gene downstream of Dmrt2a. This cytochrome was one of the few genes up-regulated after dmrt2a overexpression and the only one that was validated by the loss-of-function approach. Also, cypla is the only validated gene from the microdissected Region 2 . However, Cyp1a physiological function in the embryo remains unknown therefore, its interaction with Dmrt2a will require further analysis.

Interestingly, we validated three genes related to vascular development: $c x c 112 b$, etv2 and foxc1b. Although we lack functional data to explain the relationship between Dmrt2a and these genes, when considering $d m r t 2 a$ expression pattern within the somite, we can contemplate the hypothesis that Dmrt2a is affecting the small population of somite-derived endothelial cells [24, 25].

cxcl12b is expressed in a subset of the myotome, named endotome, which will colonise the dorsal aorta and will contribute to endothelial and vascular associated cells in zebrafish [25]. As dmrt2a is also expressed in the myotome [2], and as it acts as a transcriptional repressor, we can speculate that it could restrict $c x c l 12 b$ endotome expression domain.

etv2-expressing angioblasts (endothelial cells precursors) migrate from the lateral plate mesoderm, 
intersomitically, towards the midline, where they contribute to the dorsal aorta and posterior cardinal vein [26]. Therefore, a possible cell non-autonomous interaction between Dmrt2a and etv2 could occur at somite territory.

Foxc proteins have been previously described to interact with etv2 [21] thus, it would be worthwhile to analyse if, the somite-expressed gene foxc1b, can cooperate with etv2 downstream of Dmrt2a. Moreover in mouse, CXCL12 receptor - CXCR4 - is transcriptionally activated by FOXC1 and FOXC2 in endothelial cells [27]. Although in zebrafish, a link between Cxcl12 signalling and Foxc transcription factors has not been established, it would be interesting to evaluate if this interaction could occur.

\section{Conclusions}

In this study, we generated and characterised an inducible dmrt2a transgenic line and mutant lines for $d m r t 2 a$ and $d m r t 2 b$. Using our transgenic line we described for the first time $d m r t 2 a$ overexpression phenotype. We confirmed that the mild phenotype present in dmrt2a mutant lines is not due to a compensatory mechanism mediated by $d m r t 2 b$. Additionally, we took advantage of one of our dmrt2a mutant lines to confirm $d m r t 2 a-\mathrm{MO}$ specificity, validating this tool.

Using our tools we performed and validated a microarray experiment that allowed the identification of six genes downstream of Dmrt2a: foxj1b, $p x d c 1 b, c x c l 12 b$, etv2, foxc1b and cyp1a.

The identification of these six genes will contribute to the understanding of Dmrt2a mechanism of action during zebrafish early development.

In which embryonic domains is Dmrt2a controlling these genes, if their interaction with Dmrt2a is direct or not and, how can these genes contribute to the previously described Dmrt2a phenotypes, will be addressed in future studies.

\section{Methods}

Zebrafish lines maintenance and husbandry

All zebrafish (Danio rerio) lines used had Tubingen background and were maintained at $28{ }^{\circ} \mathrm{C}$ on a 14 -h light/10-h dark cycle. The wildtype fish used to generate the transgenic and mutant lines were obtained from Zebrafish International Resource Center (ZIRC). Adult zebrafish were only used as breeders. The embryos when collected were kept in an incubator at $28{ }^{\circ} \mathrm{C}$, under the same light/dark cycle, until the appropriate stage of development [11].

When growing a new fish line, embryos were bleached before reaching $24 \mathrm{hpf}$, as described [28]. After 5 days in the incubator at $28{ }^{\circ} \mathrm{C}, 40-50$ larvae were transferred to tanks of $3 \mathrm{l}$, where they grew during 1 month. The number of juvenile fish per litre decreased gradually until reaching adulthood. Adult breeding zebrafish (3 months old) were maintained at a density of five fish per litre.

Zebrafish were raised and maintained in a six-rack recirculating system from Tecniplast supplied by a reverse osmosis unit. Conductivity was maintained at $800 \mu \mathrm{S}$ with ocean salt (Aquarium Systems) and $\mathrm{pH}$ was maintained at 7.0, and adjusted with sodium bicarbonate (Sigma-Aldrich). Fish were fed with ZEBRAFEED (Sparos, Portugal) $<100 \mu \mathrm{m}$ (larvae), between 100 and $200 \mu \mathrm{m}$ (juvenile fish) and between 200 and $400 \mu \mathrm{m}$ (adults). Additionally, adult fish ate a meal of decapsulated Artemia (ZM Systems) per day.

All experiments were performed with embryos obtained from an outcross of homozygous progenitors, in the case of transgenic fish, and from an incross of two homozygous progenitors, in the case of mutant fish.

\section{Transgenic line generation}

To generate the $\mathrm{Tg}(\mathrm{hsp} 70: \mathrm{HA}-d m r t 2 a)$, dmrt2a cDNA with an N-terminal HA-tag was placed downstream of the $h s p 70$ heat-shock promoter in the pT2 vector (UAS-hsp70 promoter-polyA- $\gamma$-crystallin promoter-CFP). dmrt $2 a$ total RNA was extracted using TRIzol (Thermo Fisher), and cDNA was synthesised using the M-MLV Reverse Transcriptase Kit (Thermo Fisher). The transgenic construct was generated using a set of PCR reactions: (1) dmrt2a sequence was amplified using the pF-dmrt2a-HA-Nter (5'-CCAGACTACGCTTCCCTTATGACGGATCTGTCC GGCACGGAG-3') to add the C-terminal of the HA sequence to the N-terminal of $d m r t 2 a$ and a pR-dmrt2a-StuI (5'-AGGGGAAAACTGAGATTTCCGATTTAAAG

AAAGCGC-3') to add a StuI restriction site to the C-terminal of dmrt2a and, (2) using the primer pF-HA-K-ClaI (5'-CCATCGATGGCCACCATGGCTTC ATATCCTTACGATG-3') we generated the full-length HA sequence, added the Kozak sequence and the ClaI restriction site. This construct was cloned into the pGEM-T Easy Vector (Promega) and screened using the blue-white screening technique. Next, this construct and the pT2 vector were digested with $\mathrm{ClaI}$ and StuI (New England BioLabs) and gel purified using the Wizard SV Gel and PCR Clean-Up System (Promega). A ligation reaction between the ClaI-Kozak-HA-dmrt2a-StuI fragment and the pT2 vector was performed using the T4 ligase (Promega). The positive colonies were selected and the DNA purified through a Genopure Plasmid Midi Kit (Roche). 20 pg (14 ng. $\mathrm{LL}^{-1}$ ) of $\mathrm{pT} 2$ plasmid containing the hsp70:HA-dmrt2a insert were injected into one-cell stage zebrafish embryos together with $7 \mathrm{pg}\left(5 \mathrm{ng} . \mu \mathrm{L}^{-1}\right)$ of transposase mRNA.

\section{Southern blot}

Southern blot analysis was performed as described [29]. Briefly, F1 heterozygous adult transgenic fish were 
anaesthetised using Tricaine (Sigma-Aldrich). The tail fin was cut, and genomic DNA was extracted. About $10 \mu \mathrm{g}$ of DNA were digested with StuI (New England Biolabs), and the DNA fragments were separated by gel electrophoresis, at $30 \mathrm{~V}$ overnight. Afterwards, the gel was stained and washed, and the DNA transferred to a nylon membrane positively charged (Roche). To cross-link the DNA, the membrane was irradiated using UV light $(\lambda=254 \mathrm{~nm})$. The pre-hybridisation and hybridisation were performed using the ULTRAhyb buffer (Ambion). The radiolabelled probe was added at $10^{9} \mathrm{cpm} . \mathrm{mL}^{-1}$ of ULTRAhyb, and the hybridisation was carried out at $42{ }^{\circ} \mathrm{C}$ for $16-24 \mathrm{~h}$. The $\gamma$-crystallin probe was amplified from the pT2 vector using the set of primers, pFCrisSB1 (5'-ATACGACACTGCATGGATC ACCTGAAAG-3') and pRCrisSB1 (5'-CTTTACCCA AAGAGTTATCCAGCATTCC-3'), which generated a fragment of $561 \mathrm{bp}$. The PCR product was gel purified using the Wizard SV Gel and PCR Clean-Up System (Promega). The DNA was labelled with ${ }^{32} \mathrm{P}$-dATP (PerkinElmer) using the Random Primer Labelling Kit (Stratagene). The signal was detected using the Phosphoimager Cassette (Molecular Dynamics) and analysed using the Typhoon System Model 9210 (GE Healthcare Life Sciences).

\section{Heat-shock experiments}

In all heat-shock experiments, embryos were raised at $28{ }^{\circ} \mathrm{C}$. At bud-stage, embryos were placed in embryo medium $(5.02 \mathrm{mM} \mathrm{NaCl}, 0.17 \mathrm{mM} \mathrm{KCl}, 0.33 \mathrm{mM}$ $\mathrm{CaCl}_{2} .2 \mathrm{H}_{2} \mathrm{O}, 0.33 \mathrm{mM} \mathrm{MgSO} 4.7 \mathrm{H}_{2} \mathrm{O}, 10^{-5} \%$ methylene blue) pre-warmed at $39^{\circ} \mathrm{C}$, and heat-shocked for $30 \mathrm{~min}$ at the same temperature. Embryos were then collected and maintained at $28{ }^{\circ} \mathrm{C}$ until the appropriate stage of development. $\operatorname{Tg}(\mathrm{hsp} 70: \mathrm{HA}-d m r t 2 a)$ and control embryos were heat-shocked with the same procedure.

\section{Genome editing}

TALEN were designed using TAL Effector-Nucleotide Targeter (TALE-NT) 2.0 web based tools [30] together with the R-based tool developed by Jorge Velez (NICHD). The selected genomic regions for $d m r t 2 a$ and $d m r t 2 b$ were introduced in TALEN Targeter. Custom Spacer/Repeat Variable Diresidue (RVD) Lengths were used, with the spacer ranging from 14 to 17 nucleotides and the RVD lengths ranging from 16 to 21 nucleotides. The G substitute chosen was NN. The upstream base chosen to each monomer was $\mathrm{T}$.

For $d m r t 2 a$, we designed one TALEN pair with a spacer containing the ATG start codon, Pair-1 $\left(\mathrm{P}_{1}\right)$ (TCTT GCTTTACGCGGCCAGAAAATGACGGATCTGTCCG G start codon and the DM GCACGGAGTTTGAGA), and another TALEN pair further downstream, Pair-2 $\left(\mathrm{P}_{2}\right)$ (TCAGGCGGTGTTCACCGGCCCCGGAGAGGACGA
CACGGGGTCCAAAGACGACGACAAA). For $d m r t 2 b$, we designed a TALEN pair targeting the region between the ATG start codon and the DM domain, Pair-3 $\left(\mathrm{P}_{3}\right)$ (TGCGACCCACCTAGAGTGCCTGAAAGCGGAGT GGGTTGCAGACTGCGCCGGACGGGA). TALEN binding sites are in bold caps, and the spacer region is underlined. Paired Target Finder was used to check for off-targets, the Score Cut-off chosen was 3.0.

To generate the single $d m r t 2 a$ mutants $\left(d m r t 2 a^{\Delta 100}\right.$ and dmrt $2 a^{\Delta 14}$ ), $200 \mathrm{pg}$ of $\mathrm{P}_{1}$ and $200 \mathrm{pg}$ of $\mathrm{P}_{2}$ RNA were co-injected into one-cell stage embryos. To generate the single $d m r t 2 b$ mutants, $250 \mathrm{pg}$ of $\mathrm{P}_{3}$ RNA was injected into one-cell stage embryos. RNA was synthesised using mMESSAGE mMACHINE SP6 Transcription Kit (Thermo Fisher). To generate the double mutants, dmrt $2 a^{\Delta 100-/-}$ was crossed with $d m r t 2 b^{-/-}$and $d m r t 2 a^{\Delta 14-/-}$ was crossed with $d m r t 2 b^{-/-}$.

To screen the F0 mosaics and the following generations of dmrt $2 b$ single and double mutants, a High Resolution Melting screening method was developed using Corbett Rotorgene 6000 (Corbett Life Science) and Power SYBR Green PCR Master Mix (Thermo Fisher). The primers used for this method were: pF-HRMdmrt2b (5'-CACA GGTAGATGCGACCCAC-3') and pR-HRMdmrt2b (5'-C TTCATCCGTGCCCATGACC-3'). To screen $d m r t 2 a^{\Delta 100}$ and $d m r t 2 a^{\Delta 14}$ single and double mutants, a PCR followed by gel electrophoresis was used with the following primers: pFdmrt2a (5'-GACACGTTACATGCAGGAAAACA-3') and pRdmrt2a (5'-GCGGTCAGATTTGTCGTCGT-3').

\section{Morpholino microinjection}

A dmrt $2 a$ antisense morpholino targeting the ATG region (5'-AGATCCGTCATTTTCTGGCCGCGTA-3') [3, 5] and a standard control morpholino (5'-CCTCTTACC TCAGTTACAATTTATA-3') obtained from Gene Tools, were injected at $1.5 \mathrm{ng} / \mathrm{embryo}$, into one-cell stage embryos. In the morpholino validation experiments, we injected $d m r t 2 a$ morpholino in the mutant background, in the same days and using the same settings as in the wildtype counterparts, to ensure a controlled procedure.

\section{In situ hybridisation and immunohistochemistry}

Single whole-mount in situ hybridisation was performed as described [31]. Embryos were photographed with a LEICA Z6 APO stereoscope coupled to a LEICA DFC490 camera.

Digoxigenin-labelled antisense RNA probes were synthesised from DNA templates of deltaC [32], her1 [33], her7 [34], mespb [35], pcdh8 [36], myod1 [37], cb1045 [38], spaw [39], pitx2 [40], myl7 [41], cxcl12b [25], etv2 [42], foxj1b [19], foxc1b [43], dmrt2a [4], cyp1a, which was cloned in pGEM-T Easy (Promega) using the pF-cypla (5'-ATGGCTCTGACTATTCTTCCAATATTG GG-3') and pR-cypla (5'-CTAGAACCCAGGCTGTGG 
TGTGACCCGA-3') and $p x d c 1 b$, which was cloned in pGEM-T Easy (Promega) using the pF-pxdc1b (5'-ATGG CATCGGCGATTTTTGAGGGCA-3') and pR-pxdc1b (5'-AAGTCAGTTTCAAAAGGAACCAGA-3').

For HA immunohistochemistry, the embryos were fixed after heat-shock in 4\% paraformaldehyde overnight, incubated with rat anti-HA antibody (1:200, 3F10, Roche \#11867423001) followed by anti-rat Alexa Fluor 594 (1:500, Thermo Fisher \#A11007). F-actin and nuclei were detected with Alexa Fluor 488-Phalloidin (1:400, Thermo Fisher \#A12379) and DAPI $\left(10 \mu \mathrm{L} . \mathrm{mL}^{-1}\right)$, respectively. Embryos were photographed with a Zeiss LSM 510 Meta.

\section{Western blot}

Embryos were collected $1 \mathrm{~h} 30 \mathrm{~min}$ and $2 \mathrm{~h} 30 \mathrm{~min}$ after heat-shock, treated as described [44] and kept at $-70{ }^{\circ} \mathrm{C}$. The following procedure was performed as described [45]. For HA-tag detection, the membranes were incubated overnight with rabbit anti-HA antibody $\left(1: 1000, \mathrm{Y}^{-11}\right.$, sc-805, Santa Cruz). For beta-actin, we used a rabbit anti-beta-actin (1:5000, ab8227, Abcam). As secondary antibody, we used an HRP-conjugated mouse anti-rabbit IgG antibody (1:10000, sc-2357, Santa Cruz).

\section{Microarray}

Between the period of $2 \mathrm{~h}$ and $2 \mathrm{~h} 30 \mathrm{~min}$ after heat-shock, $\operatorname{Tg}($ hsp70:HA-dmrt2a) and wildtype embryos were microdissected as described [46]. Tissues from Region 1 and Region 2 (Fig. 9a, c) were kept in TRIzol (Thermo Fisher) at $-70{ }^{\circ} \mathrm{C}$. We obtained a pool of 100 pieces from Region 1 and 100 pieces from Region 2, from each condition, per replicate. Three biological replicates for each condition were used. Total RNA was extracted using TRIzol (Thermo Fisher) and further purified with RNA Clean \& Concentrator-5 kit (Zymo Research). RNA quality was assessed with an Agilent 2100 Bioanalyzer (Agilent Genomics), and all samples had a RIN value between 8.60 and 9.30. All samples were analysed with Affymetrix Zebrafish Gene 1.1 ST Array Strip (Thermo Fisher). The raw data (CEL files) were analysed with Affymetrix Expression Console Software, Affymetrix Transcriptome Analysis Console Software (Thermo Fisher) and R. Statistical analysis was performed using one-way ANOVA and False Discovery Rate (FDR) correction. Only genes with a fold change (FC) higher than 2 or lower than -2 , with $P<0.05$ and $\mathrm{FDR}<0.05$, were considered with the exception of foxc1b with a $\mathrm{FC}=-1,3, P=0.00134$ and $\mathrm{FDR}=0.103$. Gene Ontology (GO) analysis was performed using AmiGO [47] with Bonferroni correction.

\section{Quantitative RT-PCR}

Total RNA was extracted from zebrafish embryos with the appropriate stage, using TRIzol (Thermo Fisher) and further purified using the Direct-zol RNA Miniprep Kit (Zymo Research). For the evaluation of dmrt2a transcript throughout development, we used $n=30$ wildtype embryos, per replicate. For the gain-of-function experiment, we collected embryos from the $\operatorname{Tg}$ (hsp70:HA-dmrt2a) line and wildtype embryos, and performed a heat-shock (Fig. 9a). All embryos were microdissected in Region 1 and Region 2 (Fig. 9a, c). From this approach, we obtained a pool of 60 pieces from Region 1 and a pool of 60 pieces from Region 2, from each condition (experimental and control), per replicate. For the loss-of-function experiment, we injected wildtype embryos with $d m r t 2 a-\mathrm{MO}$ or control-MO, and microdissected them in Region 1 and Region 2 (Fig. 9b, c). From this approach, we obtained a pool of 46 pieces from Region 1 and a pool of 46 pieces from Region 2, from each condition (experimental and control), per replicate. For the evaluation of mutant transcripts, we used the different $d m r t 2 a$ and $d m r t 2 b$ mutant embryos and wildtype embryos, at $24 \mathrm{hpf}$, with $n=10$, per replicate. Three biological replicates were used in each experiment. cDNA was synthesised using DyNAmo cDNA Synthesis Kit (Thermo Scientific) using random hexamer primers. Quantitative PCR was performed using Corbett Rotorgene 6000 (Corbett Life Science) and Power SYBR Green PCR Master Mix (Thermo Fisher). Quantification of the relative expression was performed using the $2^{-\Delta \Delta C t}$ method. We evaluated each gene on an individual basis, between experimental and control samples. Data were analysed using a two-tailed $t$-test. The primers used are listed in Additional file 9: Table S1.

\section{Statistical analysis}

All experiments were performed with at least three biological replicates, collected from different breeders. The statistical analysis performed in the microarray experiment was a one-way ANOVA and in qPCR experiments was a two-tailed $t$-test. $P$ values of less than 0.05 were considered significant.

\section{Additional files}

\footnotetext{
Additional file 1: Figure S1. TALEN pairs. a Alignment between TALEN $\mathrm{P}_{1}$ and $d m r t 2 a$ sequence. $\mathbf{b}$ Alignment between TALEN $\mathrm{P}_{2}$ and $d m r t 2 a$ sequence. c Alignment between TALEN $P_{3}$ and $d m r t 2 b$ sequence. The start codon is underlined, and the spacer region is in lowercase. Each colour represents a different Repeat Variable Diresidue (RVD). (TIF 3783 kb)

Additional file 2: Figure S2. HA-Dmrt2a dynamics after heat-shock. Immunostaining showing the expression of the HA-Dmrt2a fusion protein at different time-points post-heat-shock. The HA-Dmrt2a protein is depicted in red, and the nucleus is depicted in blue. Min: minutes. Scale bar: $10 \mu \mathrm{m}$. (TIF 16094 kb)

Additional file 3: Figure S3. Microarray data. a, b List of all the genes obtained in the microarray with $\mathrm{FC}>2$ or $\mathrm{FC}<-2, P<0.05$ and $\mathrm{FDR}<0.05$, together with $d m r t 2 a$, foxcl $a$ and foxclb in Region 1 ( $F C=1.3, P=0.000416$, $\mathrm{FDR}=0.0677 ; \mathrm{FC}=-1.04, P=0.219, \mathrm{FDR}=0.636 ; \mathrm{FC}=-1.3, P=0.00134$,
} 
$F D R=0.103$, respectively). (a) Region 1 and (b) Region 2. Genes related to cardiac and vascular development are underlined. (TIF $679 \mathrm{~kb}$ )

Additional file 4: Figure S4. Gene Ontology (GO) analysis of the microarray data. a, $\mathbf{b}$ Most significant $\mathrm{GO}$ terms associated with the microarray data with $\mathrm{FC}>2$ or $\mathrm{FC}<-2, P<0.05$ and $\mathrm{FDR}<0.05$. (a) Region 1 and (b) Region 2. (TIF $1345 \mathrm{~kb}$ )

Additional file 5: Figure S5. $\mathrm{qPCR}$ validation of the microarray data in gain and loss-of-function experiments. a, a' qPCR validation of selected genes from the microarray in a gain-of-function approach. (a) Region 1 and $\left(\mathbf{a}^{\prime}\right)$ Region 2. $\mathbf{b}, \mathbf{b}^{\prime}$ qPCR validation of selected genes from the microarray in a loss-of-function approach using dmrt2a-MO. (b) Region 1 and $\left(\mathbf{b}^{\prime}\right)$ Region 2. ${ }^{*} P<0.05,{ }^{* *} P<0.01,{ }^{* * *} P<0.001$, ns: not significant. $P$-values were generated using a two-tailed $t$-test. (TIF $1349 \mathrm{~kb}$ )

Additional file 6: Figure S6. No misexpression was found in the validated genes after dmrt2a gain and loss-of-function experiments. A1-L2' In situ hybridisation results depicting the six genes validated in this work. A1-B2" etv2 expression pattern after injecting ctrMO (A1-A1"), dmrt2a-MO (A2-A2"), and after heat-shock in control (B1-B1") and Tg(hsp70:HA-dmrt2a) (B2-B2"). C1-D2"' foxj1b expression pattern after injecting ctrMO (C1-C1"'), dmrt2a-MO (C2-C2"'), and after heat-shock in control (D1-D1"') and Tg(hsp70:HA-dmrt2a) (D2-D2"'). E1-F2" cyp1a expression pattern after injecting ctrMO (E1-E1"), dmrt2a-MO (E2-E2"), and after heat-shock in control (F1-F1") and Tg(hsp70:HA-dmrt2a) (F2-F2"). G1-H2 cxcl12b expression pattern after injecting ctrMO (G1), dmrt2a-MO (G2), and after heat-shock in control (H1) and Tg(hsp70:HAdmrt2a) (H2). I1-J2 pxdc1b expression pattern after injecting ctrMO (I1), dmrt2a-MO (12), and after heat-shock in control (J1) and Tg(hsp70:HAdmrt2a) (J2). K1-L2' foxclb expression pattern after injecting ctrMO (K1,

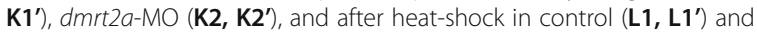
Tg(hsp70:HA-dmrt2a) (L2, L2'). After dmrt2a overexpression using $\operatorname{Tg}($ hsp70:HA-dmrt2a) we did not observe misexpression of the six validated genes. Upon comparison with dmrt2a-MO injected embryos, we observed changes in the expression levels of some genes (arrowheads), according to GPCR data. As depicted with asterisks, after dmrt2a-MO injection we observed only very subtle changes in the expression pattern of some genes, corresponding to the less affected genes, as quantified by qPCR. All embryos were collected between 3 and 4-somite stage (loss-of-function experiments) and between $2 \mathrm{~h}$ and $2 \mathrm{~h} 30 \mathrm{~min}$ after heat-shock (gain-of-function experiments). (A1-B2, C1-D2, E1-F2, G1-H2, 11-J2, K1-L2) Lateral view, anterior to the left. (A1'-B2', C1'-D2', E1'-F2') Dorsal-anterior view, anterior to the top. (A1"-B2", C1"'-D2"', E1"-F2", K1'-L2') Dorsal-posterior view, anterior to the top. (C1"-D2") Dorsal-medial view, anterior to the top. ctrMO: control morpholino. (TIF 13209 kb)

Additional file 7: Figure S7. dmrt2a expression pattern using in situ hybridisation after gain and loss-of-function experiments. A1-B2' dmrt2a expression pattern after injecting ctrMO (A1, $\left.\mathbf{A} \mathbf{1}^{\prime}\right)$, dmrtza-MO (A2, $\left.\mathbf{A} \mathbf{2}^{\prime}\right)$ and after heat-shock in control (B1, B1') and Tg(hsp70:HA-dmrt2a) (B2, B2'). C1-C3' dmrtza expression pattern in wildtype embryos ( $\left.\mathbf{C 1}^{\prime}, \mathbf{C 1}^{\prime}\right)$, in dmrt2 $a^{\Delta 100-1-} ; d_{m r t 2 b^{-1}}$ embryos injected with $\left.\mathrm{ctrMO}(\mathbf{C 2}, \mathbf{C 2})^{\prime}\right)$ and dmrt2a-MO (C3, C3'). (A1-C3) Lateral view, anterior to the left. (A1'-C3') Dorsal view, anterior to the top. All embryos were collected between 3 and 4-somite stage (loss-of-function experiments) and between $2 \mathrm{~h}$ and $2 \mathrm{~h} 30 \mathrm{~min}$ after heat-shock (gain-of-function experiments). ctrMO: control morpholino, WT: wildtype. (TIF $3087 \mathrm{~kb}$ )

Additional file 8: Figure $\mathbf{S 8}$. dmrt2a-MO is specific for the validated genes. A1-F3' In situ hybridisation results depicting the six genes validated in this work. A1-A3" etv2 expression pattern in wildtype embryos (A1-A1"), in dmrt2a $a^{\Delta 100-1-} ; d_{m r t} 2 b^{-1-}$ embryos injected with ctrMO (A2-A2") and dmrt2a-MO (A3-A3"). B1-B3"' foxj1b expression pattern in wildtype embryos (B1-B1"'), in dmrt2a $a^{\Delta 100-1-} ; d_{m r t 2 b^{-1}}$ embryos injected with ctrMO (B2-B2"') and dmrt2a-MO (B3-B3"'). C1-C3' cypla expression pattern in wildtype embryos $\left(\mathbf{C} 1, \mathbf{C 1}^{\prime}\right)$, in dmrtza $a^{\Delta 100}$ ${ }_{-{ }^{--}} ; d_{m r t 2 b^{-/-}}$embryos injected with ctrMO $\left(\mathbf{C 2}, \mathbf{C} \mathbf{2}^{\prime}\right)$ and dmrt2a-MO (C3, C3'). D1-D3 cxcl12b expression pattern in wildtype embryos (D1) in dmrt2a $a^{\Delta / 00-1-} ; d_{m r t 2 b^{-/-}}$embryos injected with ctrMO (D2) and dmrt2a-MO (D3). E1-E3 pxdclb expression pattern in wildtype embryos (E1), in dmrt2a $a^{\Delta 100-1-} ; \mathrm{dmr}^{2} 2 b^{-/-}$embryos injected with ctrMO (E2) and dmrt2a-MO (E3). F1-F3' foxc1b expression pattern in wildtype embryos
$\left(\mathbf{F 1}, \mathbf{F} 1^{\prime}\right)$, in $d m r t 2 a^{\Delta 100-/-} \cdot d m r t 2 b^{-/-}$embryos injected with ctrMO $\left(\mathbf{F} 2, \mathbf{F 2}^{\prime}\right)$ and dmrt2a-MO $\left(\mathbf{F} 3, \mathbf{F 3}^{\prime}\right)$. We did not observe obvious differences between the three different conditions evaluated. All embryos were collected between 3 and 4-somite stage. (A1-A3, B1-B3, C1-C3, D1-D3, E1-E3, F1-F3) Lateral view, anterior to the left. (A1'-A3', B1'-B3') Dorsal-anterior view, anterior to the top. (A1"-A3", B1"'-B3"', $\left.C 1^{\prime}-C 1^{\prime}, F 1^{\prime}-F 3^{\prime}\right)$ Dorsal-posterior view, anterior to the top. (B1"-B3") Dorsal-medial view, anterior to the top. ctrMO: control morpholino, WT: wildtype. (TIF $8561 \mathrm{~kb}$ )

Additional file 9: Table S1. Primers used in quantitative RT-PCR. (DOC $97 \mathrm{~kb}$ )

\section{Abbreviations}

cDNA: Complementary DNA; ctr: Control; DM: Doublesex and Mab3; DMRT: Doublesex and Mab3 related transcription factors; FC: Fold change; FDR: False discovery rate; GO: Gene ontology; HA: Haemagglutinin; hpf: Hours post-fertilisation; LRO: Left-Right Organiser; MO: Morpholino; mRNA: Messenger RNA; ns: Not significant; $P_{1}$ : TALEN pair $1 ; P_{2}$ : TALEN pair 2; $P_{3}$ : TALEN pair 3; RIN: RNA integrity number; RVD: Repeat variable diresidue; TALEN: Transcription activator-like effector nucleases; UTR: Untranslated Region; WT: Wildtype

\section{Acknowledgements}

We thank the support given by the Fish Facility and the Bioimaging Unit of Instituto de Medicina Molecular. Microarray analysis was performed by Gene Expression Unit at Instituto Gulbenkian de Ciência (Oeiras, Portugal). We are grateful to Ana Rita Grosso for her help in microarray data analysis.

\section{Funding}

This research was supported by FCT (Portugal) grant (PTDC/SAU-BID/119627/ 2010) given to L.S. L.S. was supported by an IF contract from FCT (Portugal). R.A.P. was supported by a PhD fellowship (SFRH/BD/87607/2012) from FCT (Portugal). Publication was sponsored by LISBOA-01-0145-FEDER-007391, project co-funded by FEDER through POR Lisboa 2020 - Programa Operacional Regional de Lisboa, PORTUGAL 2020 and by Fundação para a Ciência e a Tecnologia.

\section{Availability of data and materials}

The datasets supporting the conclusions of this article are available in the Gene Expression Omnibus (GEO) repository, with the accession number GSE103105 (https://www.ncbi.nlm.nih.gov/geo/).

\section{Authors' contributions}

RAP performed all the experiments, analysed the data and wrote the manuscript. JA-S designed the TALEN pairs, did the TALEN microinjections and optimised the mutant screening procedures. RL conceived the plan for the transgenic line. LS conceived the project, analysed the data and wrote the manuscript. All authors read and approved the final manuscript.

\section{Ethics approval and consent to participate}

All the procedures performed in zebrafish were done at the Instituto de Medicina Molecular's fish facility, licensed by the national General Directorate authority (DGAV - Direcção Geral de Alimentação e Veterinária) complying with the Directive 2010/63/EC (transposed to the Portuguese legislation by Decreto-Lei 113/2013) and following the Federation of European Laboratory Animal Science Associations (FELASA) guidelines and recommendations concerning laboratory animal welfare, scientific use and proper education/ training of all personnel performing animal work.

The present project was approved by the ethics committee of Centro Hospitalar Lisboa Norte and Centro Académico de Medicina de Lisboa, presided by Professor Doctor José Luis B. Ducla Soares.

\section{Competing interests}

The authors declare that they have no competing interests.

\section{Publisher's Note}

Springer Nature remains neutral with regard to jurisdictional claims in published maps and institutional affiliations. 


\section{Author details}

'Instituto de Medicina Molecular, Faculdade de Medicina, Universidade de Lisboa, Avenida Professor Egas Moniz, 1649-028 Lisboa, Portugal. ${ }^{2}$ Instituto de Medicina Molecular e Instituto de Histologia e Biologia do Desenvolvimento, Faculdade de Medicina, Universidade de Lisboa, Avenida Professor Egas Moniz, 1649-028 Lisboa, Portugal. ${ }^{3}$ Present address: Instituto Gulbenkian de Ciência, 2780-156 Oeiras, Portugal. ${ }^{4}$ Present address: CEDOC, NOVA Medical School, Universidade Nova de Lisboa, 1150-190 Lisboa, Portugal.

\section{Received: 10 October 2017 Accepted: 25 May 2018} Published online: 19 June 2018

\section{References}

1. Bellefroid EJ, Leclere L, Saulnier A, Keruzore M, Sirakov M, Vervoort M, De Clercq S. Expanding roles for the evolutionarily conserved Dmrt sex transcriptional regulators during embryogenesis. Cell Mol Life Sci. 2013; 70(20):3829-45.

2. Meng A, Moore B, Tang H, Yuan B, Lin S. A Drosophila doublesex-related gene, terra, is involved in somitogenesis in vertebrates. Development. 1999; 126(6):1259-68.

3. Saude L, Lourenco R, Goncalves A, Palmeirim I. Terra is a left-right asymmetry gene required for left-right synchronisation of the segmentation clock. Nat Cell Biol. 2005;7(9):918-20.

4. Lourenco R, Lopes SS, Saude L. Left-right function of dmrt2 genes is not conserved between zebrafish and mouse. PLoS One. 2010;5(12):e14438.

5. Matsui T, Sasaki A, Akazawa N, Otani H, Bessho Y. Celf1 regulation of dmrt2a is required for somite symmetry and left-right patterning during zebrafish development. Development. 2012;139(19):3553-60.

6. Lu C, Wu J, Xiong S, Zhang X, Zhang J, Mei J. MicroRNA-203a regulates fast muscle differentiation by targeting dmrt2a in zebrafish embryos. Gene. 2017;625:49-54

7. Zhou X, Li Q, Lu H, Chen H, Guo Y, Cheng H, Zhou R. Fish specific duplication of Dmrt2: characterization of zebrafish Dmrt2b. Biochimie. 2008; 90(6):878-87.

8. Liu S, Li Z, Gui JF. Fish-specific duplicated dmrt2b contributes to a divergent function through hedgehog pathway and maintains left-right asymmetry establishment function. PLoS One. 2009;4(9):e7261.

9. Seo KW, Wang Y, Kokubo H, Kettlewell JR, Zarkower DA, Johnson RL. Targeted disruption of the DM domain containing transcription factor Dmrt2 reveals an essential role in somite patterning. Dev Biol. 2006;290(1):200-10.

10. Sato T, Rocancourt D, Marques L, Thorsteinsdottir S, Buckingham M. A Pax3/ Dmrt2/Myf5 regulatory cascade functions at the onset of myogenesis. PLoS Genet. 2010;6(4):e1000897.

11. Kimmel CB, Ballard WW, Kimmel SR, Ullmann B, Schilling TF. Stages of embryonic development of the zebrafish. Dev Dyn. 1995:203(3):253-310.

12. Amack JD, Wang X, Yost HJ. Two T-box genes play independent and cooperative roles to regulate morphogenesis of ciliated Kupffer's vesicle in zebrafish. Dev Biol. 2007;310(2):196-210.

13. Zhang L, Hua Z, Ren J, Meng A. The nuclear localisation signal of zebrafish terra is located within the DM domain. FEBS Lett. 2001;503(1):25-9.

14. Brennan J, Norris DP, Robertson EJ. Nodal activity in the node governs leftright asymmetry. Genes Dev. 2002;16(18):2339-44.

15. Noel ES, Verhoeven M, Lagendijk AK, Tessadori F, Smith K, Choorapoikayil S, den Hertog J, Bakkers J. A nodal-independent and tissue-intrinsic mechanism controls heart-looping chirality. Nat Commun. 2013;4:2754

16. Blum M, Feistel K, Thumberger T, Schweickert A. The evolution and conservation of left-right patterning mechanisms. Development. 2014 141(8):1603-13.

17. Coschigano KT, Wensink PC. Sex-specific transcriptional regulation by the male and female doublesex proteins of Drosophila. Genes Dev. 1993;7(1):42-54

18. Murphy MW, Sarver AL, Rice D, Hatzi K, Ye K, Melnick A, Heckert LL, Zarkower D, Bardwell VJ. Genome-wide analysis of DNA binding and transcriptional regulation by the mammalian Doublesex homolog DMRT1 in the juvenile testis. Proc Natl Acad Sci U S A. 2010;107(30):13360-5.

19. Tian T, Zhao L, Zhang M, Zhao X, Meng A. Both foxj1a and foxj1b are implicated in left-right asymmetric development in zebrafish embryos. Biochem Biophys Res Commun. 2009;380(3):537-42.

20. De Val S, Chi NC, Meadows SM, Minovitsky S, Anderson JP, Harris IS, Ehlers ML, Agarwal P, Visel A, Xu SM, et al. Combinatorial regulation of endothelial gene expression by ets and forkhead transcription factors. Cell. 2008;135(6): 1053-64.

21. Veldman MB, Lin S. Etsrp/Etv2 is directly regulated by Foxc1a/b in the zebrafish angioblast. Circ Res. 2012;110(2):220-9.

22. Teasdale RD, Collins BM. Insights into the PX (phox-homology) domain and SNX (sorting nexin) protein families: structures, functions and roles in disease. Biochem J. 2012;441(1):39-59.

23. Lodish H, Berk A, Zipursky SL, Matsudaira P, Baltimore D, Darnell J. 2000. Molecular cell biology. 4th edition. New York: W. H. Freeman; 2000. Section 17.3, overview of the secretory pathway. Available from: https://www.ncbi. nlm.nih.gov/books/NBK21471/.

24. Buckingham M, Mayeuf A. Skeletal muscle development. In: Muscle: Fundamental Biology and Mechanisms of Disease Boston. Boston, Waltham: Academic Press; 2012. p. 749-62.

25. Nguyen PD, Hollway GE, Sonntag C, Miles LB, Hall TE, Berger S, Fernandez KJ, Gurevich DB, Cole NJ, Alaei S, et al. Haematopoietic stem cell induction by somite-derived endothelial cells controlled by meox1. Nature. 2014; 512(7514):314-8

26. Kohli V, Schumacher JA, Desai SP, Rehn K, Sumanas S. Arterial and venous progenitors of the major axial vessels originate at distinct locations. Dev Cell. 2013;25(2):196-206.

27. Hayashi H, Kume T. Forkhead transcription factors regulate expression of the chemokine receptor CXCR4 in endothelial cells and CXCL12-induced cell migration. Biochem Biophys Res Commun. 2008;367(3):584-9.

28. Zebrafish: A Practical Approach., vol. 261. Oxford and New York: Oxford University Press; 2002.

29. Southern E. Southern blotting. Nat Protoc. 2006;1(2):518-25.

30. Cermak T, Doyle EL, Christian M, Wang L, Zhang Y, Schmidt C, Baller JA, Somia NV, Bogdanove AJ, Voytas DF. Efficient design and assembly of custom TALEN and other TAL effector-based constructs for DNA targeting Nucleic Acids Res. 2011;39(12):e82.

31. Thisse $C$, Thisse B. High-resolution in situ hybridisation to whole-mount zebrafish embryos. Nat Protoc. 2008;3(1):59-69.

32. Jiang YJ, Aerne BL, Smithers L, Haddon C, Ish-Horowicz D, Lewis J. Notch signalling and the synchronisation of the somite segmentation clock. Nature. 2000:408(6811):475-9.

33. Holley SA, Geisler R, Nusslein-Volhard C. Control of her1 expression during zebrafish somitogenesis by a delta-dependent oscillator and an independent wave-front activity. Genes Dev. 2000;14(13):1678-90.

34. Oates AC, Ho RK. Hairy/E(spl)-related (her) genes are central components of the segmentation oscillator and display redundancy with the Delta/notch signaling pathway in the formation of anterior segmental boundaries in the zebrafish. Development. 2002;129(12):2929-46.

35. Sawada A, Fritz A, Jiang YJ, Yamamoto A, Yamasu K, Kuroiwa A, Saga Y, Takeda $H$. Zebrafish Mesp family genes, mesp-a and mesp-b are segmentally expressed in the presomitic mesoderm, and Mesp-b confers the anterior identity to the developing somites. Development. 2000;127(8):1691-702.

36. Yamamoto A, Amacher SL, Kim SH, Geissert D, Kimmel CB, De Robertis EM. Zebrafish paraxial protocadherin is a downstream target of spadetail involved in morphogenesis of gastrula mesoderm. Development. 1998; 125(17):3389-97.

37. Weinberg ES, Allende ML, Kelly CS, Abdelhamid A, Murakami T, Andermann P, Doerre OG, Grunwald DJ, Riggleman B. Developmental regulation of zebrafish MyoD in wild-type, no tail and spadetail embryos. Development. 1996;122(1):271-80

38. Lee HC, Tseng WA, Lo FY, Liu TM, Tsai HJ. FoxD5 mediates anterior-posterior polarity through upstream modulator Fgf signaling during zebrafish somitogenesis. Dev Biol. 2009;336(2):232-45.

39. Hashimoto H, Rebagliati M, Ahmad N, Muraoka O, Kurokawa T, Hibi M, Suzuki T. The Cerberus/Dan-family protein Charon is a negative regulator of nodal signaling during left-right patterning in zebrafish. Development. 2004 131(8):1741-53.

40. Essner JJ, Amack JD, Nyholm MK, Harris EB, Yost HJ. Kupffer's vesicle is a ciliated organ of asymmetry in the zebrafish embryo that initiates left-right development of the brain, heart and gut. Development. 2005;132(6):1247-60.

41. Yelon D, Horne SA, Stainier DY. Restricted expression of cardiac myosin genes reveals regulated aspects of heart tube assembly in zebrafish. Dev Biol. 1999:214(1):23-37.

42. Schindler YL, Garske KM, Wang J, Firulli BA, Firulli AB, Poss KD, Yelon D. Hand 2 elevates cardiomyocyte production during zebrafish heart development and regeneration. Development. 2014;141(16):3112-22. 
43. Kim AD, Melick CH, Clements WK, Stachura DL, Distel M, Panakova D, MacRae C, Mork LA, Crump JG, Traver D. Discrete notch signaling requirements in the specification of hematopoietic stem cells. EMBO J. 2014:33(20):2363-73.

44. Westerfield $\mathrm{M}$. The zebrafish book. A guide for the laboratory use of zebrafish (Danio rerio). 4th ed. Eugene: University of Oregon Press; 2000

45. Green MR, Sambrook J. Molecular cloning: A laboratory manual. 3rd ed. New York: Cold Spring Harbor Laboratory Press; 2001.

46. Picker A, Roellig D, Pourquie O, Oates AC, Brand M. Tissue micromanipulation in zebrafish embryos. Methods Mol Biol. 2009;546:153-72.

47. AmiGO: http://amigo.geneontology.org/amigo. Accessed October 2015.

Ready to submit your research? Choose BMC and benefit from:

- fast, convenient online submission

- thorough peer review by experienced researchers in your field

- rapid publication on acceptance

- support for research data, including large and complex data types

- gold Open Access which fosters wider collaboration and increased citations

- maximum visibility for your research: over $100 \mathrm{M}$ website views per year

At BMC, research is always in progress.

Learn more biomedcentral.com/submissions 